\title{
Article
}

\section{Lipid-hydrogel films for sustained drug release}

Kulkarni, Chandrashekhar Vishwanath, Moinuddin, Zeinab, PatilSen, Yogita, Littlefield, Rowan and Hood, Mitesh

Available at http://clok.uclan.ac.uk/11406/

Kulkarni, Chandrashekhar Vishwanath ORCID: 0000-0002-5621-4791, Moinuddin, Zeinab, Patil-Sen, Yogita, Littlefield, Rowan and Hood, Mitesh (2015) Lipid-hydrogel films for sustained drug release. International Journal of Pharmaceutics, 479 (2). pp. 416-421. ISSN 0378-5173

It is advisable to refer to the publisher's version if you intend to cite from the work. http://dx.doi.org/10.1016/j.ijpharm.2015.01.013

For more information about UCLan's research in this area go to http://www.uclan.ac.uk/researchgroups/ and search for <name of research Group>.

For information about Research generally at UCLan please go to http://www.uclan.ac.uk/research/

All outputs in CLoK are protected by Intellectual Property Rights law, including Copyright law. Copyright, IPR and Moral Rights for the works on this site are retained by the individual authors and/or other copyright owners. Terms and conditions for use of this material are defined in the policies page.

\section{CLoK}

Central Lancashire online Knowledge www.clok.uclan.ac.uk

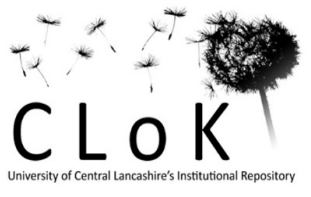




\section{Lipid-Hydrogel Films for Sustained Drug Release}

2 Chandrashekhar V. Kulkarni ${ }^{a^{*}}$, Zeinab Moinuddin ${ }^{a \dagger}$, Yogita Patil-Sen ${ }^{a, c \dagger}$, Rowan Littlefield ${ }^{b \dagger}$

3 and Mitesh Hood ${ }^{a, c}$

$4{ }^{a}$ Centre for Materials Science, School of Forensic and Investigative Sciences, University of

5 Central Lancashire, Preston PR1 2HE, United Kingdom.

$6 \quad{ }^{b}$ Department of Chemistry, Stetson University, 421 N. Woodland Blvd. DeLand, Florida

7 32723, USA.

$8{ }^{c}$ School of Pharmacy and Biomedical Sciences, University of Central Lancashire, Preston PR1

9 2HE, United Kingdom.

10 † Share equal authorship

11 *Corresponding Author: Email: cvkulkarni@uclan.ac.uk, Tel: +44-1772-89-4339, Fax:

$12+44-1772-89-4981$. Centre for Materials Science, School of Forensic and Investigative

13 Sciences, University of Central Lancashire, Preston PR1 2HE, United Kingdom.

\section{Keywords}

15 nanostructured lipid particles; nanomedicine; sustained release; lipid-hydrogel matrix; lipid

16 self-assembly, hydrogel films; drug nanocarriers

\section{Abbreviations ${ }^{1}$}

18 Abstract

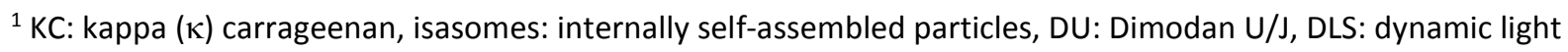
scattering
} 
1 We report a hybrid system, fabricated from nanostructured lipid particles and

2 polysaccharide based hydrogel, for sustained release applications. Lipid particles were

3 prepared by kinetically stabilizing self-assembled lipid nanostructures whereas the hydrogel

4 was obtained by dissolving kappa-carrageenan (KC) in water. The drug was incorporated in

5 native as well as lipid particles loaded hydrogels, which upon dehydration formed thin

6 films. The kinetics of drug release from these films was monitored by UV-Vis spectroscopy

7 while the films were characterized by Fourier Transform Infra-Red (FTIR) spectroscopy

8 and small angle $\mathrm{x}$-ray scattering techniques. Pre-encapsulation of a drug into lipid particles

9 is demonstrably advantageous in certain ways; for instance, direct interactions between KC

10 and drug molecules are prohibited due to the mediation of hydrophobic forces generated by

11 lipid tails. Rapid diffusion of small drug molecules from porous hydrogel network is

12 interrupted by their encapsulation into rather large sized lipid particles. The drug release

13 from the lipid-hydrogel matrix was sustained by an order of magnitude timescale with

14 respect to the release from native hydrogel films. These studies form a strong platform for

15 the development of combined carrier systems for controlled therapeutic applications. 


\section{Introduction}

2 Nanostructured lipid particles, also called 'isasomes’(Yaghmur et al., 2005) (internally selfassembled somes-particles) have demonstrably proved their potential as delivery vehicles for

4 active molecules such as drugs, proteins and genes (Angelova et al., 2011; Han et al., 2010;

5 Hirlekar et al., 2010; Yaghmur and Glatter, 2009). These particles are simply oil-in-water

6 (O/W) type emulsions (Fig.1) but the fascination lies in their interior, which is comprised of

7 highly organized self-assembled lipid nanostructure that can be cubic or hexagonal

8 (Gustafsson et al., 1996, 1997); corresponding particles are called ‘cubosomes’ and

9 'hexosomes'. The internal nanostructure can be further varied to lamellar, sponge and reverse micellar phases (Kulkarni, 2012). Advantages of dispersed lipid particles with respect to their bulk (equilibrium phases) counterparts involve much lower viscosity, ability to withstand extremely high levels of dilutions, easier to manipulate and availability of much larger surface area (Kulkarni and Glatter, 2012). Apparent viscosity of a dispersion of lipid particles can be altered further by varying concentration of dispersed phase up to a certain extent ( $70 \%$ in some cases (Salentinig et al., 2008)) but a greater control can be achieved by forming thick, durable hydrogels in the aqueous region (continuous phase) (Guillot et al., 2009; Tomsic et al., 2009). Hydrogels can be formed from natural polymers such as cellulose, starch, carrageenan and chitosan which are often used in food and cosmetic

19 industries as thickeners or viscosity enhancers (Kadajji and Betageri, 2011; Shit and Shah, 2014). In addition to their non-toxicity, biocompatibility and biodegradability, these polymers exhibit good swelling properties thanks to their three-dimensional cross-linked hydrophilic networks that are capable of holding large volumes of water (typically in the range of 30 to $90 \%$ ) (Shit and Shah, 2014). The highly porous structure of these hydrogel networks facilitates the encapsulation of various bioactive molecules such as drugs, proteins 
1 and DNA. Therefore they are being widely explored for controlled delivery applications

2 (Kadajji and Betageri, 2011; Lee et al., 2013).

3 Reversible incorporation of nanostructured lipid particles in hydrogels was first

4 demonstrated by Tomsic et al. (Tomsic et al., 2009) and Guillot et al. (Guillot et al., 2009).

5 The concept was further extended towards formation of dry films (Kulkarni et al., 2011).

6 Nanostructured lipid particles could be retrieved back upon rehydration and re-dissolution of

7 gel films. Important properties of lipid particles-the size and the nanostructure were

8 preserved consequent to the above hysteresis (Kulkarni et al., 2011). We have employed this

9 approach to develop a hybrid system for drug delivery. The hybrid system was formulated

10 by loading the drug in the dispersion of nanostructured lipid particles and mixing it with the

11 hydrogel; the mixture was then poured in custom made moulds and dehydrated to form drug 12 loaded film.

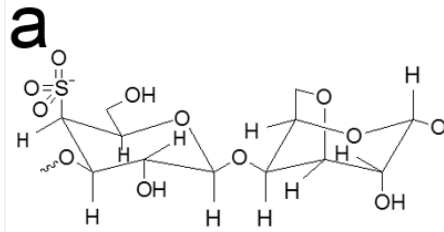

-Carrageenan (KC)

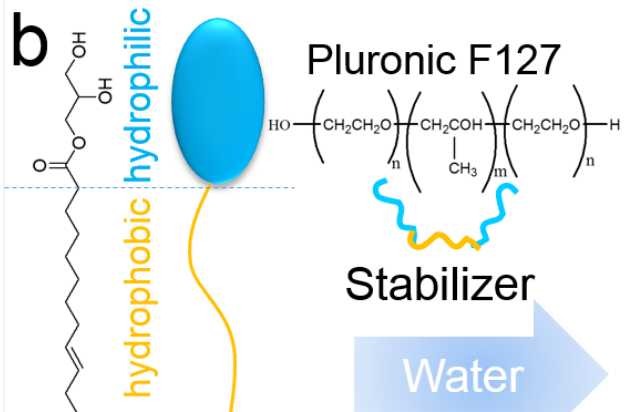

Ultrasonication

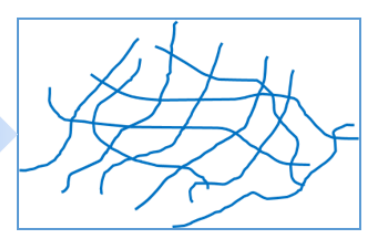

Hydrogel
Native hydrogel
O/W emulsion

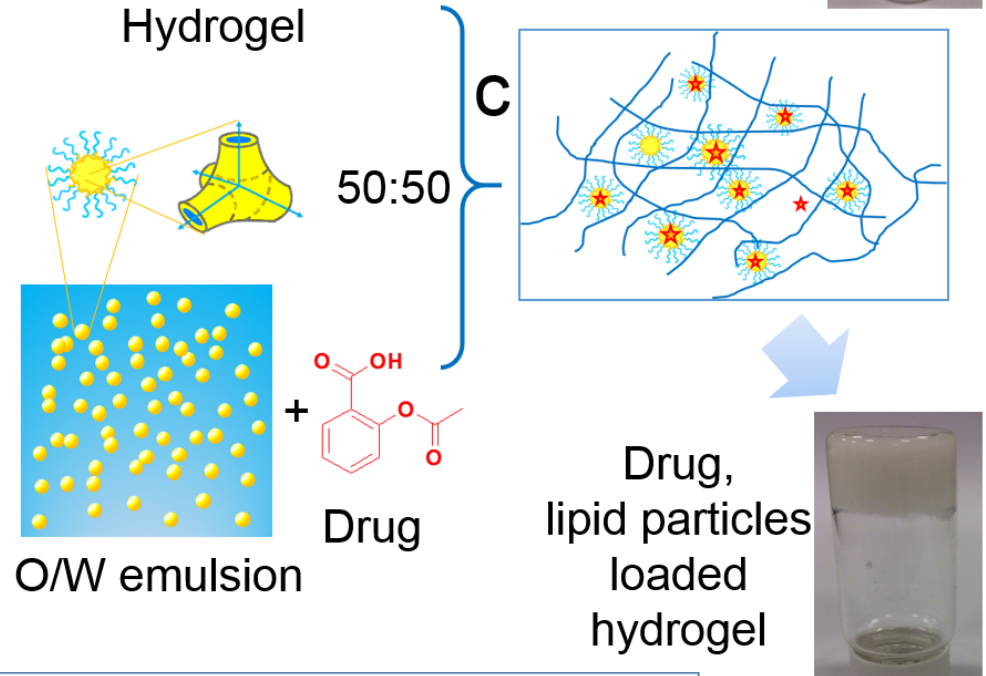

Lipid molecule

Self-assembled lipid nanostructure $\kappa$-Carrageenan

\section{ᄂ} - 
Fig.1 a) Structural unit of KC forming cross-linked hydrogel network which looks stiff and transparent in upside down glass vial. b) Amphiphilic lipid molecule forms lipid cubic phase inside the particles stabilized by pluronic F127 stabilizer upon ultrasonication. These particles are then loaded with drug and mixed with KC hydrogel in equal ratio to form opaque hydrogel as shown in c). Schematic diagrams are used for ease of understanding the constituents and their location.

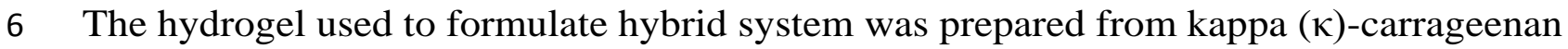
(KC). It is a high molecular weight anionic sulphated linear polysaccharide of D-galactose and 3,6-anhydro D-galactose (Fig. 1a) originated from red seaweed of the Rhodophyceae

9 family (Prajapati et al., 2014). The KC hydrogel forms at room temperature (sol-gel transition at $28.4{ }^{\circ} \mathrm{C}$ in cooling direction (Tomsic et al., 2008)) and melts above $60{ }^{\circ} \mathrm{C}$. Although the gel formed from mere $2 \% \mathrm{KC}$ is rather stiff (storage modulus G' $\sim 10^{4} \mathrm{~Pa}$, Note: storage modulus is a property of viscoelastic materials and can be used to estimate gel strength) (Tomsic et al., 2008), it is a 'physical gel' which imbibes large amount of water and constitutes irregular pores ranging up to $600 \mu \mathrm{m}$ size (Daniel-da-Silva et al., 2007). Because of these characteristics, KC pellets employed for drug delivery applications were found to disintegrate faster in aqueous media resulting in rapid release of drugs (Kranz et al., 2009). Kranz et al. showed that about half of the drug gets released from KC pellets in first 10 minutes while the release is almost complete within an hour. Other disadvantages of pure KC system are that they require expensive drying methods and they also suffer from stability problems for sensitive drugs (Bornhöft et al., 2005). However, modified forms of KC, its amalgamation with other polymers and encapsulation of micro/nanoparticles have shown to be more effective ways to use them as drug carriers (Li et al., 2014). In this report we have encapsulated drug loaded internally-self assembled soft lipid particles within a KC hydrogel 
1 form but also release it in an efficient and sustained manner. Systematic characterization of

2 this and relevant control systems along with time-resolved studies of drug release process

3 form central part of this work.

\section{2. Materials and Methods}

\section{$5 \quad 2.1$ Materials}

6 Commercial grade Dimodan-U/J (DU) was a gift from Danisco, Denmark. It is a distilled

7 glyceride comprising $96 \%$ monoglycerides (Fig. 1b) and the rest are diglycerides and free

8 fatty acids. Two major monoglyceride components in DU are linoleate (62 \%) and oleate (25

$9 \%$ \%). Hence the hydrophobic part of DU mainly contains C18 chains (91\%). The triblock

10 copolymer Pluronic ${ }^{\circledR}$ F127 (PEO99 -PPO67-PEO99) (Fig. 1b) was purchased from Sigma-

11 Aldrich, UK. It was used as an emulsion stabilizer. The hydrogelling agent, KC (Fig. 1a)

12 was obtained from Fluka Biochemika, Switzerland. Aspirin was purchased from Sigma-

13 Aldrich, UK. It is a salicylate drug (Fig. 1b) used mainly as analgesic and antipyretic agent.

14 All the chemicals were used as received without any further purification. DU was stored

15 below $4{ }^{\circ} \mathrm{C}$ whereas the other materials were kept at room temperature. Water used during

16 entire study was purified using Barnstead Nanopure, Thermoscientific (USA).

$17 \quad 2.2$ Preparation and Loading of Nanostructured Lipid Particles Nanostructured lipid particles

18 were prepared according to the published method using slightly different compositions and

19 parameters (Yaghmur et al., 2005). $500 \mathrm{mg}$ of molten lipid (DU) was added to the $20 \mathrm{ml}$

20 glass vial and topped with the stabilizer solution (prepared separately by dissolving $0.5 \%$

21 pluronic F127 in $100 \mathrm{ml}$ water) to give a total weight of $10 \mathrm{gm}$. The mixture was

22 ultrasonicated (ULT065 from Ultrawave, Cardiff, UK) for 10 minutes with a continuous

23 pulse at $35 \%$ power. Samples became hot due to ultrasonication, hence were allowed to 
cool to room temperature before further use. Drug was loaded by stirring 5 mg of aspirin (in

2 powder form) into the above solution for about 15 min to ensure the drug was dissolved into

3 the "milky" emulsion (of nanostructured lipid particles).

\section{$4 \quad 2.3$ Loading KC hydrogel}

$54 \% \mathrm{KC}$ solution was prepared by stirring $\mathrm{KC}$ powder in $9.6 \mathrm{ml}$ water on hot plate at $60{ }^{\circ} \mathrm{C}$

6 for around 20 minutes. The molten, pourable solution was combined with an equal amount

7 of drug loaded nanostructured lipid particle dispersion and gently stirred (for 15-20 minutes)

8 while heating until visual homogenization (Kulkarni et al., 2011). Native KC gel (without

9 nanostructured lipid particles) was prepared by simply stirring 5 mg aspirin with 2 \% KC

10 solution at $60^{\circ} \mathrm{C}$.

\section{$11 \quad 2.4 \mathrm{UV}-\mathrm{Vis}$ Spectroscopy}

12 UV-Visible spectroscopy (UV-3600, Shimadzu, UV-Vis NIR Spectrophotometer, Japan)

13 was used to monitor drug release from drug loaded films in pure water. Using $4 \mathrm{ml}$ quartz

14 cuvettes the spectra in the range of wavelength 200-700 nm were recorded at every 30 or 60

15 min. Typical release experiments were performed for about 24 hrs and a few long runs for

1642 hrs. All experiments were repeated at least three times.

\section{$17 \quad 2.5$ Small Angle X-ray Scattering}

18 Small angle x-ray scattering measurements (1.2-10 degrees) on dehydrated samples and dry

19 films were performed using D2 Phaser (Bruker Instruments, UK). Typical settings of 0.1

$20 \mathrm{~mm}$ slit and $0.1 \mathrm{~mm}$ knife edge distance were used to acquire 1-dimensionsal data

21 (integrated using DIFFRAC.SUITE over intensity) with LYNXEYE ${ }^{\mathrm{TM}}$ detector.

22 Measurements were performed at room temperature $\left(20^{\circ} \mathrm{C}\right)$. Typical measurements of 20

23 minutes were sufficient to produce useful data. 


\subsection{Fourier Transform Infra-Red Spectroscopy}

2 A Thermoscientific Nicolet IR 200, FT-IR model was used to carry out the ATR-IR

3 experiments. Spectra were generated for each type of film used as well as for each of their

4 constituent components in the range of frequencies $400-4000 \mathrm{~cm}^{-1}$. All measurements were 5 conducted at room temperature.

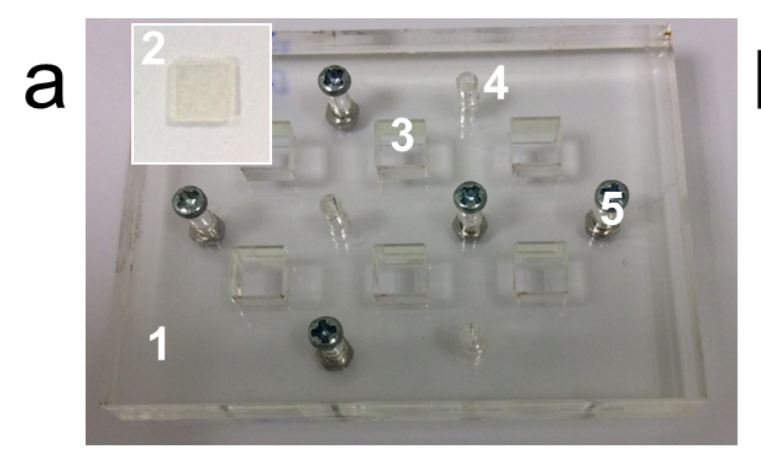

1 mould, 2 ready film, 3 square well, 4 hole for screw, 5 screw
6

$7 \quad$ Fig.2 a) Perspex 2-part mould designed in-house to produce 6 films at a time. Inset shows the dry

8 film loaded with nanostructured lipid particles and a drug, which was placed at the bottom of

9 cuvette as shown in b). The drug release in pure water as a function of time was recorded by UV-

10 spectroscopy at regular intervals.

\section{3. Results and discussion}

\section{$12 \quad 3.1$ Preparation of Hydrogel Films for Drug Delivery}

13 Molten hydrogels (see section 2.3 for more details) were poured into custom designed (in-

14 house) moulds (Fig. 2a) to fabricate films. Six square holes of 6.0x6.0x1.2 mm dimensions

15 were drilled by laser-cutting in a $1.2 \mathrm{~mm}$ thick Perspex sheet which was screwed to another

16 similar sheet (Fig 2a) to construct well-like architectures. Above well dimensions were 
1 chosen to produce films that can fully swell in aqueous media without virtually touching to

2 the cuvette walls. Hydrogel films were formed after $42-48$ hrs of drying in air (lost $98 \pm 1 \%$

3 water from gel), whereas semi-dried films were prepared by drying the gels in humid

4 atmosphere for $24-30$ hrs (lost $85 \pm 2 \%$ water from gel). The two part mould attachment

5 served for easy removal of dried films. Using a flat-faced spatula, individual films were

6 carefully immersed in pure water (at the bottom of $4 \mathrm{ml}$ quartz cuvette). UV-Vis

7 measurements were started immediately (Fig. 2b) and spectra were collected at regular

8 intervals at room temperature.
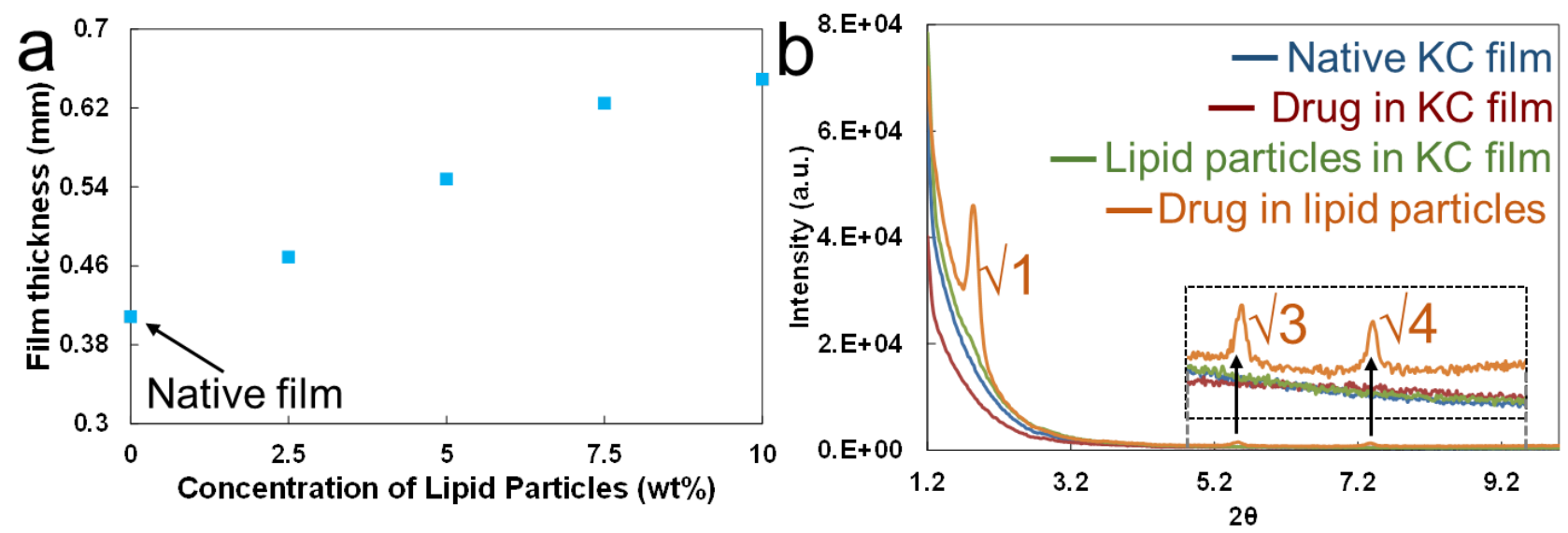

9

Fig. 3 a) Film thickness was increased linearly until loading of $7.5 \%$ nanostructured lipid particles which seem to stabilize at $10 \%$ concentration. This means more drug can be loaded in these films owing to the availability of more lipid particles without extreme change in the film dimensions. b) Small angle x-ray scattering patterns displaying typical upturn at low angles for hydrogel network. Nanostructure of lipid particles was converted from original cubic (Guillot et al., 2006) to hexagonal (characteristic indexing as $1, \sqrt{3}, \sqrt{4}$ ) due to addition of a drug.

\subsection{Structure and Properties of Hydrogel Films}

Dry films of native KC were very thin (average thickness of $\sim 0.4 \mathrm{~mm}$ ), transparent and brittle while films loaded with nanostructured lipid particles were less transparent and thick; the thickness increased linearly with the amount of loading until $7.5 \mathrm{wt} \%$ dispersed phase 
1 (Fig. 3a). The transparency is partly lost due to scattering from large lipid particles. The

2 average particle size of nanostructured lipid particles was found in the range of 2-5 $\mu \mathrm{m}$

3 (measured by dynamic light scattering, see SI Fig. SI1). Films loaded with lipid particles

4 were rather elastic compared to native KC films, which could be attributed to the

$5 \quad$ viscoelastic and sticky nature of the lipid constituent.

6 A pink tinge was seen for native $\mathrm{KC}$ films upon drug loading while films loaded with

7 nanostructured lipid particles did not show detectable pink tinge. Nevertheless, the drug

8 loading into lipid particles could be confirmed by the transformation of an internal self-

9 assembly of lipid particles. The original bicontinuous cubic Pn3m nanostructure (Guillot et

10 al., 2006) observed for pure lipid molecules (in water) was modulated to the hexagonal

11 nanostructure due to incorporation of a drug as verified by small angle $\mathrm{x}$-ray scattering (Fig.

12 3b). This aspect turns out to be beneficial because the hexagonal phase is essentially

13 constituted of water filled cylinders holding the drug for long lengths of time whereas for the

14 bicontinuous cubic phase, because of its open channel network, it is presumed to release the

15 drug at much higher rates (Phan et al., 2011). The influence of nanostructure-type on the

16 release rates has been demonstrated by a few researchers working on similar lipid systems

17 (Chemelli et al., 2012; Fong et al., 2009; Phan et al., 2011). Typical upturns at low angles

18 (without other distinguishable peaks) observed for KC films (Fig. 3b) could be ascribed to

19 large hydrogel networks (Tomsic et al., 2008).

\section{3.3 Drug Protection by Nanostructured Lipid Particles}

21 The Fourier Transform Infra-Red (FTIR) spectrum for drug-aspirin (red curve in Fig. 4)

22 shows anticipated signals including a sharp peak at $1604 \mathrm{~cm}^{-1}(\mathrm{C}=\mathrm{C}$ aromatic stretch), a

23 signal at $1677 \mathrm{~cm}^{-1}$ (conjugated acid $\mathrm{C}=\mathrm{O}$ stretch), a peak at $1747 \mathrm{~cm}^{-1}$ (ester $\mathrm{C}=\mathrm{O}$ stretch)

24 and a broad peak around $\sim 2800 \mathrm{~cm}^{-1}$ (acid O-H stretch). Several peaks for KC, as supported 
1 by literature studies (Pereira et al., 2009), could also be identified from signals at $841 \mathrm{~cm}^{-1}$

2 (corresponding to D-galactose-4-sulfate), $918 \mathrm{~cm}^{-1}$ (corresponding to 3,6-anhydro-D-

3 galactose), $1219 \mathrm{~cm}^{-1}$ (sulphate ester), and a broad peak at $3336 \mathrm{~cm}^{-1}$ (alcohol stretch).

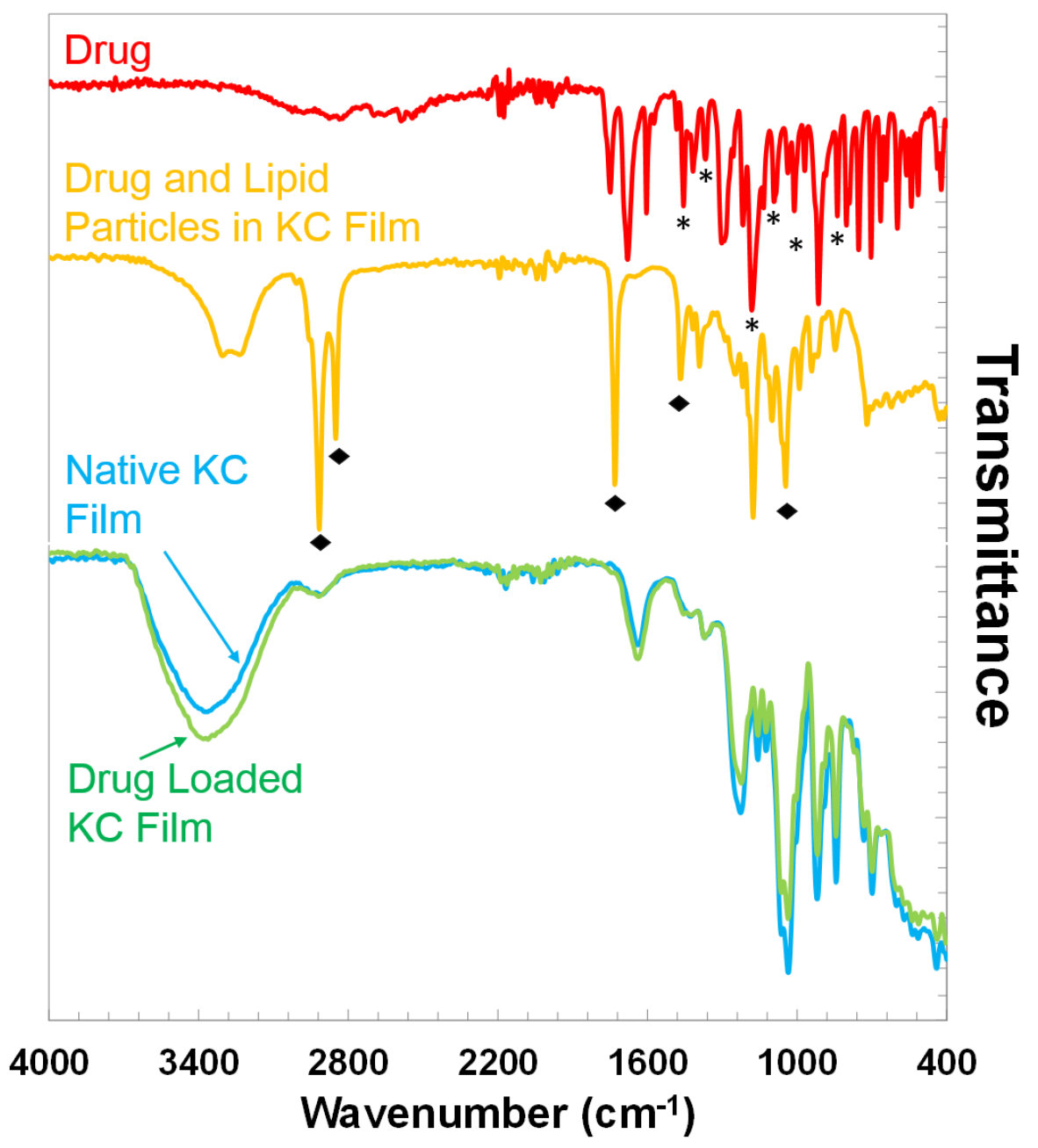

6 Fig. 4 FTIR spectra demonstrate that drug (aspirin) signals were retained after its loading into KC film together

7 with nanostructured lipid particles while there was no measureable evidence of drug loading in the native KC

8 films. Signals for lipid are shown by $\bullet$ sign (see SI Fig. SI2 for lipid only spectrum) and aspirin peaks retained

9 after loading are indicated by * sign in a drug spectra.

10 An examination of the drug loaded KC film spectrum (Fig. 4) reveals that it is practically

11 indistinguishable from the native $\mathrm{KC}$ film spectrum. Furthermore, no signals from aspirin 
1 are obviously identifiable within the drug loaded KC film spectrum. Several reasons could

2 be postulated to reveal the underlying mechanism of masking of drug signals by KC signals;

3 for instance, a) the concentration of a drug loaded in $10 \mathrm{gm}$ of hydrogel solution (used to

4 prepare films) was mere $5 \mathrm{mg}$ whereas the concentration of kappa-carrageenan (KC) was as

5 high as $200 \mathrm{mg}, \mathrm{b})$ some of the chemical groups/bonds are commonly found in both - drug

6 and KC chemical structures and c) there are strong chances of hydrogen bonding and

7 electrostatic interactions between these molecules which further mask the IR band resolution

8 However, the acquisition of a slight pinkish hue and detectable UV-Vis signals during

9 release studies imply that drug was successfully entrapped in the KC film.

10 Physico-chemical interactions between drug and KC network were also apparent from the

11 UV-Vis studies. The reference UV-Vis spectrum of aspirin showed distinct peaks at $225 \mathrm{~nm}$

12 and $275 \mathrm{~nm}$ (SI Fig. SI3) whereas these peaks were shifted to higher wavelengths in case of

13 released aspirin from native and lipid particles-loaded hydrogel films (see SI Fig. SI3). The

14 peak-shifts could be attributed to the chromophoric interactions between functional groups

15 of the components. However, these aspects were not studied in much depth here as the focus

16 of the current report is towards introducing a hybrid drug carrier system and establishing a

17 method to produce prototype for drug delivery applications.

18 In case of drug+lipid particles loaded KC films, a small acid/ester stretch at $1727 \mathrm{~cm}^{-1}$, an 19 alkyl C-H stretches at $2854 \mathrm{~cm}^{-1}$ and $2915 \mathrm{~cm}^{-1}$ and a broad alcohol stretch at $3363 \mathrm{~cm}^{-1}$

20 represent lipid signals (spectra of lipid only and drug loaded lipid particles are provided in SI

21 Fig. SI2 for reference). In addition, some discernible aspirin signals were also observed

22 (indicated by * sign in Fig. 4). Here, the drug was preloaded into lipid particles followed by

23 their incorporation into the hydrogel matrix. The repulsion between hydrophobic lipid tails

24 (of inverse lipid nanostructures) and KC strands is presumed to avoid the masking of aspirin 
1 signals by KC signals. It simply means that the lipid molecules act as a barrier for

2 electrostatic and hydrogen bonding interactions between aspirin and KC structures thereby

3 effectively protecting the structural form of a drug as opposing to the native KC films. This

4 drug pre-encapsulation, in fact, was advantageous for sustaining the release as discussed

5 below.

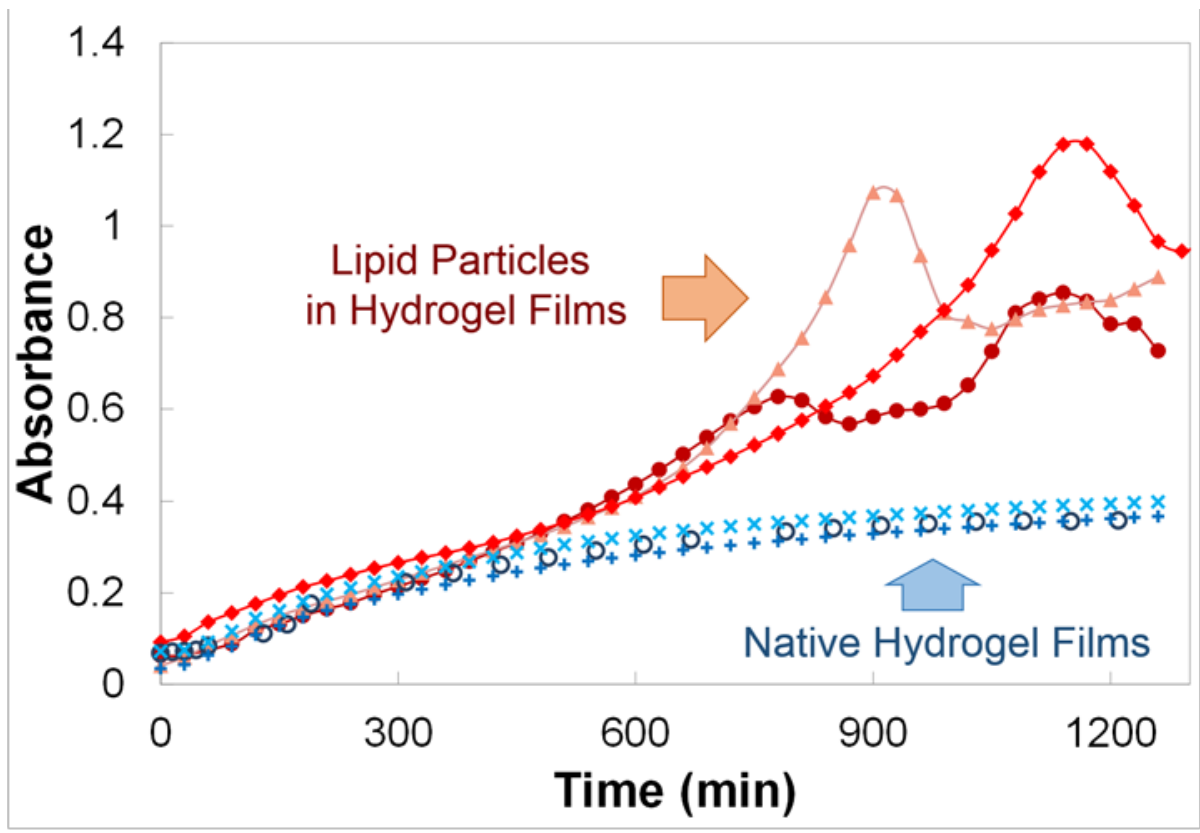

$7 \quad$ Fig.5 Drug release from native hydrogel films $(x,+$ and $o)$ stabilized in early hours whereas lipid particles loaded films $(\bullet, \mathbf{\square}$ and $\mathbf{\Lambda})$ sustained drug for a longer length of time. Large changes in absorbance values in case of films with lipid particles as compared to native films (qualitatively) represent release of more drug from lipid particles loaded films. Individual curves represent a different set of experiments performed under same conditions. Noisy curves after initial peak (after 700-800 min) could be due to possible disintegration of films to release nanostructured lipid particles

12 passing randomly across the beam.

\subsection{Drug Release from Semi-dried Films}

14 Films dried for 24-30 hrs in humid atmosphere were rather soft and more hydrated than those prepared in dry atmosphere (for 42-48 hrs). Drug release from these semi-dried films was monitored by time-resolved collection of UV-spectra similar to Fig 2b. A fixed value of 
1 wavelength was determined by Gaussian fit to the broad peak around 255-320 nm.

2 Absorbance values at this wavelength $(294 \mathrm{~nm})$ were plotted against experimental time as

3 shown in Fig. 5. Three curves (indicated by $x,+$ and o) demonstrate that the drug release

4 from native KC films was gradual in first 3-4 hrs and it stabilized soon after that. Release

5 from lipid loaded films was practically similar in early hours but it was clearly faster after

6 about 10-12 hrs (shown by $\bullet$, $\mathbf{a}$ and $\mathbf{\Lambda}$ ). This can be attributed to the dissolution of hydrogel

7 network followed by the disintegration of the whole film. Latter leads to a discharge of

8 entrapped lipid particles, which presumably pass across the UV-Vis beam randomly as

9 reflected in the noisy data after a peak around 700-800 min. Visible changes occurring with

10 the film were tracked by imaging it at various time intervals as shown in Fig. 6c. 

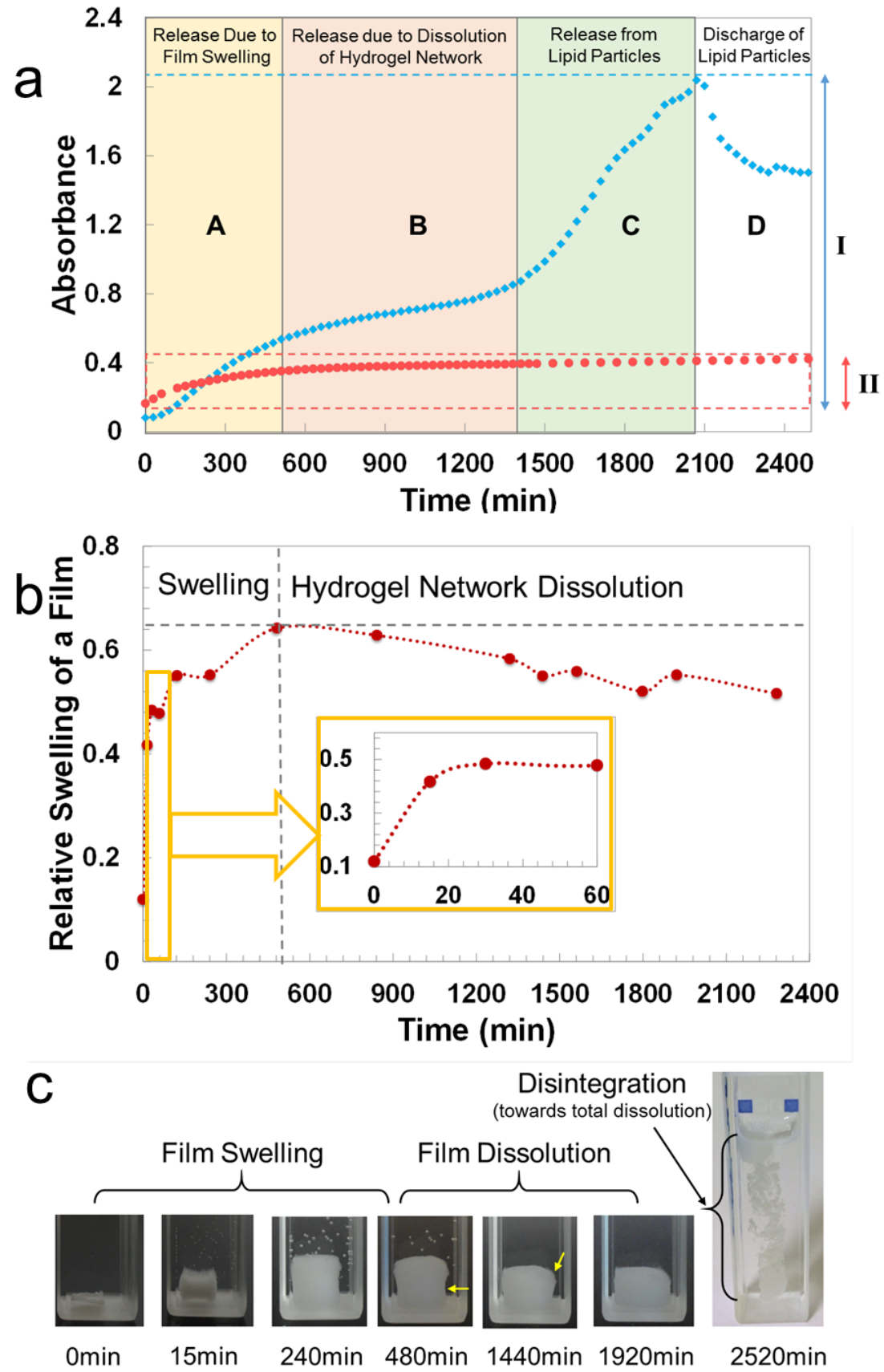

$2 \quad$ Fig. 6 a) Drug release studied for 48 hrs from fully dried native and lipid particles loaded hydrogel films. Shaded

3 regions (A, B, and C) indicate the possible mechanism of drug release from hydrogel films while the noisy data due to discharge of lipid particles is shown in D. The release from native film only occurs during early hours, which stabilizes

5 in about 600 mins (shown by $\bullet$ ). Moreover, the amount of drug released is far too less (low absorbance values) as

6 compared to the total release from lipid loaded films (shown with $\bullet$ ) as depicted by I and II. b) Relative swelling of a

7 drug+lipid particle loaded film (thickness of the film normalized by cuvette-bottom length) practically matches with 
suggested mechanism in a). c) Images captured at various time intervals apparently reflect possible physical processes associated with hydrogel films during drug release studies. Yellow arrows indicate shape changes due to dissolution of hydrogel network.

\section{$4 \quad 3.5$ Understanding the Processes Responsible for Drug Release}

5 Although the drug release curves obtained for semi-dried and fully dried films virtually

6 followed same patterns, their absolute times differed by a few hrs (Figs. 5 and 6). Semi-dried

7 films disintegrated about 900 min faster than fully dried ones, which was also the case for

8 rapid drug release prior to this step.

$9 \quad$ Different drug release rates and release times could underlay different physical processes

10 as described below. Initial rapid release (within first $10 \mathrm{hrs}$ ) could be due to swelling of a

11 film (Fig 6). During this time the dehydrated hydrogel network absorbs a maximum amount

12 of water, thereby untightening of the hydrogel network takes place. This facilitates the

13 release of entrapped drug from the hydrogel network (Region A in Fig. 6a). The swollen

14 network starts dissolving in water causing gradual drug release in the next 15 hrs (Region B

15 in Fig. 6a). This slow-down could be due to hindrance caused by lipid particles. A rapid

16 release of film content after $\sim 24$ hrs could be attributed to the release from lipid particles

17 (Region C in Fig. 6a) as their pores (aqueous channels of internally self-assembled

nanostructure) open up due to the complete dissolution of the hydrogel network surrounding them. This can be validated from the fact that the rate of drug release (slope of 0.0014) from mere lipid particles (investigated independently see SI Fig. SI4) and the release in the

21 Region C (slope of 0.0018) were quite comparable. The amount of drug released from lipid

22 loaded films was about an order of magnitude higher than the drug released from native

23 films (arbitrated from change in the initial absorbance values) as indicated by I and II in Fig.

24 6. These attributes along with FTIR results (section 2.3) convincingly prove that the 
1 efficiency of drug release from hybrid system was higher than in pure system, presumably

2 because some of the drug seized by KC and drug interactions (in native films) was free to be

3 released from the hybrid system due to lipid intervention. In late stages of dissolution, the

4 hydrogel network plausibly becomes sparse enough to discharge the entrapped lipid particles

5 leading to the noisy data after 2050 min (Region D in Fig 6). However, noticeable

6 disintegration of the film was only captured on camera at 2520 min (Fig 6c).

\section{4. Conclusions}

8 We have presented a novel application of lipid particles-loaded hydrogel films in the field of

9 drug delivery. As the main aim of the current work was to demonstrate the applicability of

10 the lipid-hydrogel films, we did not emphasize on the structural form and activity of the drug

11 in this system. However, it is clear that the encapsulation of drug molecules in lipid particles

12 is advantageous, in many ways. For instance, the drug release timescale was prolonged by

13 almost an order of magnitude as compared to the release from KC films; in addition, it was

14 released in more efficient manner (more drug was released). Furthermore, lipid particles

15 practically improve the stability of the drug which appears to have lost due to possible

16 bonding with KC network in native films. We were able to recognize the physical processes

17 responsible for differential release rates of drugs at various timescales. The lipid-hydrogel

18 composite system, presented here, finds an enormous potential for development of

19 therapeutic carriers in a range of pharmaceutical and cosmetic applications.

\section{Acknowledgements}

22 We highly appreciate support of Francis Neale from the School of Art, Design and

23 Performance, University of Central Lancashire for fabrication of moulds. We thank Nicholas 
P. Gaunt for DLS measurements. ZM and RL would like to acknowledge UCLan’s URIS-

22014 Scheme for financial support.

\section{References}

4 1. Angelova, A., Angelov, B., Mutafchieva, R., Lesieur, S., Couvreur, P., 2011. Self-Assembled Multicompartment Liquid Crystalline Lipid Carriers for Protein, Peptide, and Nucleic Acid Drug

$6 \quad$ Delivery. Acc. Chem. Res. 44, 147-156.

7 2. Bornhöft, M., Thommes, M., Kleinebudde, P., 2005. Preliminary assessment of carrageenan as

8 excipient for extrusion/spheronisation. Eur. J. Pharm. Biopharm. 59, 127-131.

9 3. Chemelli, A., Maurer, M., Geier, R., Glatter, O., 2012. Optimized Loading and Sustained

10 Release of Hydrophilic Proteins from Internally Nanostructured Particles. Langmuir 28, 167881116797.

4. Daniel-da-Silva, A., Lopes, A., Gil, A., Correia, R., 2007. Synthesis and characterization of porous $\kappa$-carrageenan/calcium phosphate nanocomposite scaffolds. J Mater Sci 42, 8581-8591.

5. Fong, W.K., Hanley, T., Boyd, B.J., 2009. Stimuli responsive liquid crystals provide 'on-demand' drug delivery in vitro and in vivo. J. Control. Release 135, 218-226.

6. Guillot, S., Moitzi, C., Salentinig, S., Sagalowicz, L., Leser, M.E., Glatter, O., 2006. Direct and indirect thermal transitions from hexosomes to emulsified micro-emulsions in oil-loaded monoglyceride-based particles. Colloids Surf., A. 291, 78-84.

7. Guillot, S., Tomsic, M., Sagalowicz, L., Leser, M.E., Glatter, O., 2009. Internally self-assembled particles entrapped in thermoreversible hydrogels. J. Colloid Interface Sci. 330, 175-179.

8. Gustafsson, J., Ljusberg-Wahren, H., Almgren, M., Larsson, K., 1996. Cubic Lipid-Water Phase Dispersed into Submicron Particles. Langmuir 12, 4611-4613. 
1 9. Gustafsson, J., Ljusberg-Wahren, H., Almgren, M., Larsson, K., 1997. Submicron Particles of

2 Reversed Lipid Phases in Water Stabilized by a Nonionic Amphiphilic Polymer. Langmuir 13, 6964-6971.

4 10. Han, S., Shen, J.Q., Gan, Y., Geng, H.M., Zhang, X.X., Zhu, C.L., Gan, L., 2010. Novel vehicle based on cubosomes for ophthalmic delivery of flurbiprofen with low irritancy and high bioavailability. Acta Pharmacol Sin 31, 990-998.

11. Hirlekar, R., Jain, S., Patel, M., Garse, H., Kadam, V., 2010. Hexosomes: A Novel Drug Delivery System. Curr. Drug Delivery 7, 28-35.

9 12. Kadajji, V.G., Betageri, G.V., 2011. Water Soluble Polymers for Pharmaceutical Applications. Polymers 3, 1972-2009.

13. Kranz, H., Jürgens, K., Pinier, M., Siepmann, J., 2009. Drug release from MCC- and carrageenan-based pellets: Experiment and theory. Eur. J. Pharm. Biopharm 73, 302-309.

14. Kulkarni, C.V., 2012. Lipid crystallization: from self-assembly to hierarchical and biological ordering. Nanoscale 4, 5779-5791.

15. Kulkarni, C.V., Glatter, O., 2012. Hierarchically Organized Systems Based on Liquid Crystalline Phases, in: Garti, N. (Ed.), Self-Assembled Supramolecular Architectures: Lyotropic Liquid Crystals. John Wiley \& Sons, Inc.

16. Kulkarni, C.V., Tomšič, M., Glatter, O., 2011. Immobilization of Nanostructured Lipid Particles in Polysaccharide Films. Langmuir 27, 9541-9550.

17. Lee, S.C., Kwon, I.K., Park, K., 2013. Hydrogels for delivery of bioactive agents: A historical perspective. Adv. Drug Deliv. Rev. 65, 17-20.

18. Li, L., Ni, R., Shao, Y., Mao, S., 2014. Carrageenan and its applications in drug delivery. 
1 19. Pereira, L., Amado, A.M., Critchley, A.T., van de Velde, F., Ribeiro-Claro, P.J.A., 2009.

2 Identification of selected seaweed polysaccharides (phycocolloids) by vibrational spectroscopy (FTIR-ATR and FT-Raman). Food Hydrocolloids 23, 1903-1909.

20. Phan, S., Fong, W.-K., Kirby, N., Hanley, T., Boyd, B.J., 2011. Evaluating the link between selfassembled mesophase structure and drug release. Int. J. Pharm. 421, 176-182.

21. Prajapati, V.D., Maheriya, P.M., Jani, G.K., Solanki, H.K., 2014. Carrageenan: a natural seaweed polysaccharide and its applications. Carbohydr Polym 105, 97-112.

8 22. Salentinig, S., Yaghmur, A., Guillot, S., Glatter, O., 2008. Preparation of highly concentrated nanostructured dispersions of controlled size. J. Colloid Interface Sci. 326, 211-220.

23. Shit, S.C., Shah, P.M., 2014. Edible Polymers: Challenges and Opportunities. Journal of Polymers 2014, 13.

24. Tomsic, M., Guillot, S., Sagalowicz, L., Leser, M.E., Glatter, O., 2009. Internally self-assembled

$$
\text { thermoreversible gelling emulsions: ISAsomes in methylcellulose, kappa-carrageenan, and }
$$
mixed hydrogels. Langmuir 25, 9525-9534.

25. Tomsic, M., Prossnigg, F., Glatter, O., 2008. A thermoreversible double gel: Characterization of a methylcellulose and [kappa]-carrageenan mixed system in water by SAXS, DSC and rheology. J. Colloid Interface Sci. 322, 41-50.

26. Yaghmur, A., de Campo, L., Sagalowicz, L., Leser, M.E., Glatter, O., 2005. Emulsified Microemulsions and Oil-Containing Liquid Crystalline Phases. Langmuir 21, 569-577.

27. Yaghmur, A., Glatter, O., 2009. Characterization and potential applications of nanostructured aqueous dispersions. Adv Colloid Interface Sci 147-148, 333-342. 